\title{
Whole-grain intake as a marker of healthy body weight and adiposity
}

\author{
Janice I Harland ${ }^{1, *}$ and Lynne E Garton ${ }^{2}$ \\ ${ }^{1}$ HarlandHall Associates, The Stables, Ranbury Ring, London Road, Poulton, Cirencester, GL7 5HN, UK: \\ ${ }^{2}$ Alimenta Ltd, Aylesbury, UK
}

Submitted 18 April 2007: Accepted 9 September 2007: First published online 16 November 2007

\begin{abstract}
Objective: To review evidence relating to the consumption of whole grains and healthy body weight (BW).

Design: Systematic review and analysis of observational studies reporting wholegrain consumption and measures of BW and adiposity, including the effect on macronutrient intakes and lifestyle factors.

Setting: Medline and other databases were searched for the period 1990 to 2006 to produce a full reference list; observational studies were retained for further analysis if they included an appropriate control group and reported whole-grain intake and body mass index (BMI) or a measure of adiposity.

Subjects: Fifteen trials were identified which included data from 119829 male and female subjects aged 13 years and over.

Results: The combined and weighted mean difference in BMI from 15 studies representing 20 treatment groups $(n=119829)$ using a random-effects model was $0.630 \mathrm{~kg} / \mathrm{m}^{2}$ lower when high versus low whole-grain intake was compared, $P<0.0001$ (95\% confidence interval (CI) $0.460,0.800 \mathrm{~kg} / \mathrm{m}^{2}$ ). In high consumers, adiposity assessed as waist circumference was reduced by $2.7(95 \%$ CI $0 \cdot 2,5 \cdot 2)$ $\mathrm{cm}, P=0.03$ (six data sets, $n=4178$ ) or as waist:hip ratio by 0.023 (95\% CI 0.016 , 0.030 ), $P<0.0001$ (four data sets, $n=20147$ ). Higher intake of whole grains led to increased dietary fibre intake $(9 \mathrm{~g}, P<0.01)$, while total and saturated fat intakes decreased by $11 \mathrm{~g}$ and $3.9 \mathrm{~g}$, respectively.

Conclusion: A higher intake of whole grains (about three servings per day) was associated with lower BMI and central adiposity. In addition, people who consume more whole grains are likely to have a healthier lifestyle as fewer of them smoke, they exercise more frequently and they tend to have lower fat and higher fibre intakes.
\end{abstract}

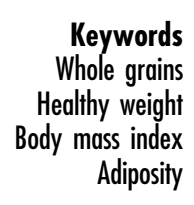

The challenge of obesity to the health of Western society has been clearly identified by the World Health Organization $(\mathrm{WHO})^{(1)}$, which also recognises that maintenance of a healthy weight is in itself as large a challenge as obesity. In the UK, the Department of Health's publication, Choosing Health: Making Healthy Choices Easier, aimed to improve the understanding of the increasing rise in the overweight and obese population in the UK and from this numerous strategies and networks are being developed to reduce the prevalence of an obese population $^{(2)}$. Guidance on the prevention, identification, assessment and management of overweight and obesity in adults and children was published by the National Institute for Clinical Excellence (NICE) in December 2006. The first two NICE dietary guidelines for healthy weight maintenance recommend ${ }^{(3)}$ :

- Base meals on starchy foods such as potatoes, bread, rice and pasta, choosing whole grains wherever possible;
- Eat plenty of fibre-rich foods - such as oats, beans, peas, lentils, grains, seeds, fruit and vegetables, as well as whole-grain bread and brown rice and pasta.

The recent Dietary Guidelines for Americans ${ }^{(4)}$ state that 3 or more ounce equivalents of whole-grain products should be consumed per day (with the rest of the recommended grains coming from enriched or wholegrain products) and whole-grain intake is also emphasised in advice given by $\mathrm{WHO}^{(5)}$. Its guidelines include promoting healthy behaviours to encourage, motivate and enable individuals to lose weight by eating more fruit and vegetables, as well as nuts and whole grains. In summarising the totality of evidence of factors that may protect or be causative in obesity and weight management, WHO adopted an assessment of data which described evidence as being convincing, probable, possible and insufficient. It concluded that convincing evidence existed to suggest that high intake of energy-dense, 
micronutrient-poor foods was associated with obesity. It promoted the intake of foods low in energy density, i.e. energy-dilute foods such as fruit, vegetables and wholegrain cereals that are high in dietary fibre and water. The relationship between dietary fibre and body weight (BW) has been explored in two reviews of randomised trials $^{(6,7)}$, which concluded that the majority of studies show that a high intake of non-starch polysaccharides (NSP dietary fibre) promotes weight loss. While this relationship between fibre and BW has been extensively studied, the relationship between whole grains and BW has not received the same degree of scientific scrutiny.

It has been argued that the beneficial role of whole grains in the diet exceeds the provision of dietary fibre $^{(8-10)}$, but its specific role and the mechanisms involved in weight management have been little researched. A brief review of whole-grain consumption and weight gain conducted in $2003^{(11)}$ concluded that epidemiological data were sparse. Indeed, evidence from randomised controlled trials is also limited. To this end in the UK the Food Standards Agency has commissioned two large intervention studies which aim to provide mechanistic evidence for whole grains and cardiovascular disease, but also to underpin public health strategies related to whole grains ${ }^{(8)}$.

The awareness of the potential public health benefits from the inclusion of whole grains in the diet was raised with the publication of health claims in the USA ${ }^{(12)}$, UK and Sweden ${ }^{(13)}$ and has led to more information about whole grains being published in recently reported epidemiological studies.

The objective of the present study was to review the scientific literature relating to whole-grain intake and its effect on healthy BW and adiposity, exploring the weight and strength of the evidence that exists. Clearly important public health recommendations are already being made with regard to whole-grain consumption; its inclusion in the diet offers an attractive and food-based dietary strategy for targeting the whole population. However, it is important to better understand the scientific evidence that supports its inclusion in diets for a healthy BW and whether it can be promoted as a marker of healthy BW.

\section{Materials and methods}

\section{Searching}

Computerised scientific publication databases were searched. The search was focused on Medline (www.ncbi.nlm.nih.gov/entrez/query.fcgi) for the period 1990 to December 2006 and complemented by searches in EMBASE and hand search of key papers and publications. In addition, the reference lists in identified papers were scrutinised for further studies. Search terms were 'wholegrain or whole grain' and 'obesity or body weight or BMI or body fat' in Medline, and with the addition of 'body measures' in EMBASE. The search was limited to human studies and those published in English or with a summary in English. The initial search was conducted by the British Library and complemented by hand searches conducted by the authors.

\section{Identification and selection of studies}

The main objective was to systematically review observational studies published since 1990 in which BW and whole-grain intake of volunteers had been assessed by suitable methods. The aim was to compare a nil or low consumption of whole grains with a much higher intake, preferably about three servings per day. In studies reporting whole-grain intake in grams, the equivalent intake was approximately $48 \mathrm{~g} / \mathrm{d}$.

The broad criteria for study inclusion were the following.

- Original epidemiology research: cohort, cross-sectional or case studies in humans reported in full in peerreviewed journals.

- Clear definition and measurement of whole grains.

- Use of suitable measures of BW maintenance: for example, body mass index (BMI), waist circumference (WC) or waist:hip ratio (WHR).

- Reports that measured a range of whole-grain intakes in comparable populations - preferably a group with no or very low whole-grain intake that could be compared with a population consuming about three servings per day or more - and that employed a suitable measure of BW or adiposity.

Duplicates of the same cohort or study were excluded, with the most recent report included. This review and analysis were conducted following the principles for systematic review of observational studies detailed by Stroup et al. ${ }^{(14)}$. The review was conducted by both authors and differences resolved by detailed discussion.

\section{Data abstraction and quality assessment}

The key characteristics of the studies were abstracted and the findings collated, which included: identification of the number of volunteers, gender, age, location and duration of study, measurements of BW or adiposity, macronutrient content of the diet (where available), definition of 'whole-grain' and method of assessment of intake. The data abstracted were subject to quality assessment in three main areas:

- recruitment and flow of subjects through the study;

- dietary assessment including definition of 'wholegrain';

- treatment and reporting of data.

Analysis of appropriate subgroups resulted from this assessment. Where data were not available in the 
published paper the authors were contacted requesting the necessary detail.

\section{Definition of whole-grain foods}

Definition of 'whole-grain' is problematic, with definitions varying in different countries. The US Food and Drug Administration permits health claims on foods that contain at least $51 \%$ of whole grains (including milled products of whole grains). The most commonly used definition in research defines whole-grain intake from products containing $25 \%$ or more of whole grains ${ }^{(15)}$. However, using data derived from the National Diet and Nutrition Survey (NDNS) in young people aged 4-18 years, it has been shown that defining whole-grain foods as those containing at least $51 \%$ of whole grains underestimates whole-grain intake by $28 \%{ }^{(16)}$; lowering the threshold to $\geq 25 \%$ content of whole grains to define whole-grain foods reduces underestimation to $15 \%$, when compared with a definition based on foods containing $\geq 10 \%$.

In addition to inconsistent definition of whole-grain intake, servings frequently used as a measure in foodfrequency questionnaires (FFQs) also vary in amount. Consequently, it was considered to be impossible to confine the review to a single definition; instead attention was focused on reports where a wide range of wholegrain intake was reported between high and low consumers. Some reports refer to intake of servings and others to intake in grams; where possible results are reported on the basis of servings per day. If total wholegrain servings were not reported, it was considered essential that either bread or breakfast cereal intake should be assessed as they are major sources of whole grains in both the $\mathrm{UK}^{(16-18)}$ and the US diet ${ }^{(19)}$.

\section{Measures of weight maintenance}

In the area of weight management, the usual measure to assess risk is BMI. It is defined as BW divided by the square of height and is reported in units of $\mathrm{kg} / \mathrm{m}^{2}$. Obesity is defined as BMI $\geq 30 \mathrm{~kg} / \mathrm{m}^{2}$, overweight as BMI $\geq 25$ to $<30 \mathrm{~kg} / \mathrm{m}^{2}$ and healthy weight as $\mathrm{BMI}<25 \mathrm{~kg} / \mathrm{m}^{2}$ (reference 1). In earlier studies WHR was used to provide an indication of central adiposity, although a better and now more frequently used measure of adiposity is WC. Three categories each for men and women relate WC to obesity; for men these are $<94 \mathrm{~cm}$, $\geq 94$ to $<102 \mathrm{~cm}$ and $\geq 102 \mathrm{~cm}$, and for women these are $<80 \mathrm{~cm}, \geq 80$ to $<88 \mathrm{~cm}$ and $\geq 88 \mathrm{~cm}$. These equate to the healthy, overweight and obese categories of BMI. In the UK the higher two categories have been designated 'action levels' 1 and 2, corresponding respectively to slightly increased and substantially increased risk of chronic conditions such cardiovascular disease and diabetes ${ }^{(20)}$.

\section{Statistical analysis}

The key outcomes reported were mean values for BMI, WHR or WC from the lowest and highest whole-grain intake groups (usually about three servings per day). For computation of the pooled effect, each study was assigned a weight consisting of the reciprocal of its variance. Estimates of mean difference and 95\% confidence interval (CI) were calculated by using a random-effects model. The assumption of heterogeneity implied by the use of random-effects models is plausible because of the differences in amounts of whole-grain intake, study populations, study duration, initial BMI and the presence of other covariates. To explore the possible influence of these covariates further, pre-specified subgroup analysis was conducted based on quality assessment of studies and biological plausibility. Finally, to estimate potential publication bias, we plotted the standard error of the studies against their corresponding effect size to produce funnel plots. All statistical analyses were conducted using Comprehensive Meta Analysis statistical software package (Biostat, Englewood, NJ, USA).

A secondary outcome reported herein compares key aspects associated with a healthy lifestyle in the low and high consumption populations. The main lifestyle factors reported were smoking, activity level, macronutrient intake and alcohol intake. Mean values and standard error of the difference were calculated and subject to a Student's $t$ test assuming population variances not to be equal.

\section{Results}

\section{Trial flow}

Figure 1 outlines the results from the reported selection process. The search identified 115 papers; when abstracts were scanned and exclusion criteria applied, this resulted in 26 potentially relevant studies. After reading the full articles, 22 studies remained that excluded duplicate reports of the same cohort at different periods of followup. Exclusion of a further three reports that included inadequate detail or insufficient range of whole-grain intakes and three non-epidemiological studies resulted in 15 retained reports ${ }^{(21-35)}$, two of which related to data not published in full (Good et al. ${ }^{(34)}$ was a conference abstract, Thane et $a l .{ }^{(35)}$ was an Epublication ahead of print at the time of the search and analysis; both sets of authors provided further details as a personal communication). Details of the 15 retained papers containing cross-sectional data or reports of cohorts from which cross-sectional data were extracted are shown in Table 1.

\section{Study characteristics}

Each report contained data from a single study with one exception, the study of Thane et $a l^{(35)}$, which included two separate sources, specifically the 1986/87 


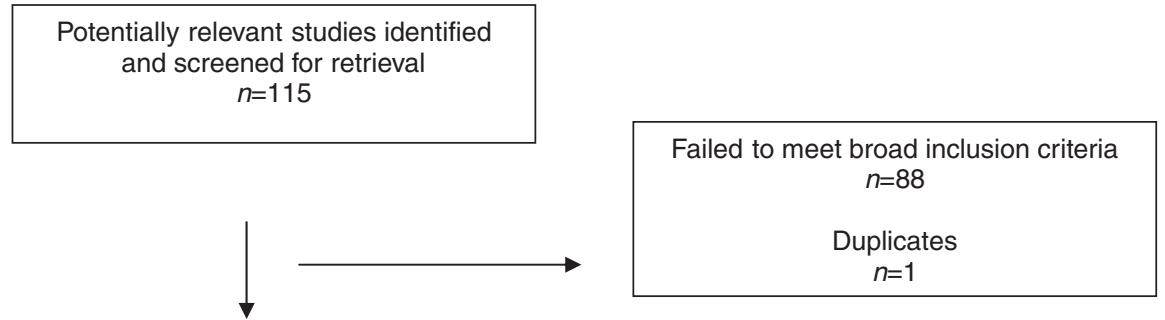

Studies retrieved for detailed evaluation $n=26$

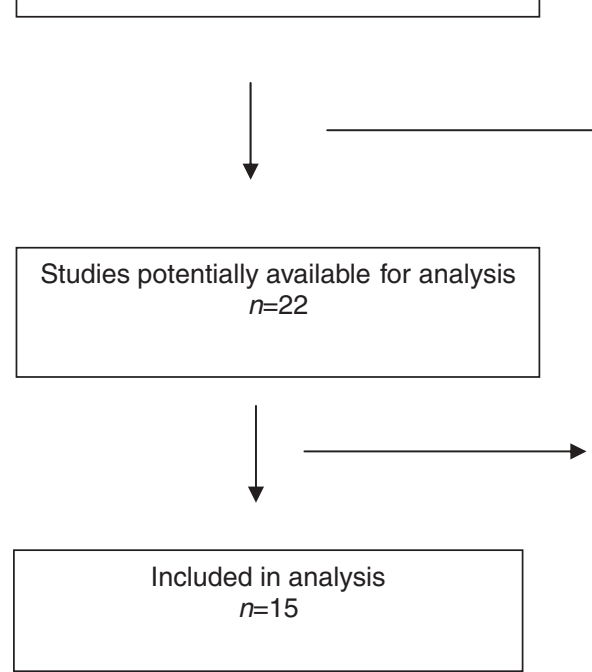

Fig. 1 Systematic review - flow of studies

and 2000/01 NDNS. Information was presented by gender separately in three papers ${ }^{(32,34,35)}$, with the result that 20 data pairs were available for analysis representing 119829 subjects. Table 1 provides details of the main characteristics of the studies including BMI, WC and WHR.

All studies apart from two ${ }^{(22,34)}$ reported energy intake. The key nutrient combinations reported were fat and/or saturated fat and dietary fibre intake, reported in nine studies $^{(23-29,31,33)}$. The main lifestyle factors reported were the proportion of subjects currently smoking and exercising regularly and alcohol consumption.

\section{Analysis of main outcomes}

The mean difference in BMI between the lowest and high intakes of whole grains for each of the 20 data pairs is shown in Fig. 2. The combined and weighted mean difference in BMI using a random-effects model resulted in a reduction of $0.630 \mathrm{~kg} / \mathrm{m}^{2}$ when high whole-grain intake was compared with low or no whole-grain intake, $P<0.0001$ (95\% CI $0.460,0.800 \mathrm{~kg} / \mathrm{m}^{2}$ ).

Adiposity was assessed by comparison of the difference in either mean WC or WHR in the lowest and highest groups of whole-grain intake, see Fig. 3. WC (six data pairs, $n=4178)$ was reduced by $2 \cdot 7(95 \%$ CI $0 \cdot 2,5 \cdot 2) \mathrm{cm}$,
$P=0.03$, in high consumers of whole grains; WHR (four data pairs, $n=20147$ ) was reduced by 0.023 (95\% CI 0.016, 0.030), $P<0 \cdot 0001$.

\section{Subgroup analysis}

The introduction of more stringent exclusion criteria resulted in the exclusion of studies not reporting any dietary information (three data pairs) or where the intake of whole grains was less than about three servings per day $(48 \mathrm{~g} / \mathrm{d})$. Additionally, attention was focused on gender and the effect computed for males and females separately, or study location where studies conducted in the USA were compared with those conducted in Europe. The results of subgroup analysis are shown in Table 2.

The exclusion of studies which did not include dietary information or focusing on studies where intake was less than about three servings had little effect on the difference in BMI. There was a small gender difference; BMI reduction in women was marginally greater than in men. Geographic location had a small effect on the reduction in BMI, with BMI loss being marginally higher in the USA compared with Europe.

The mean nutrient intakes for the low and high wholegrain data sets are shown in Table 3 . Higher whole-grain 
Table 1 Details of the cohorts and cross-sectional data included in the present systematic review and analysis

\begin{tabular}{|c|c|c|c|c|c|c|c|c|c|c|c|c|c|c|c|}
\hline Study & $\begin{array}{l}\text { Sex, Age } \\
\text { range } \\
\text { (years) }\end{array}$ & Country & $n$ & Trial description & $\begin{array}{l}\text { No data } \\
\text { group }\end{array}$ & WG definition & $\begin{array}{l}\text { WG intake } \\
\text { determination }\end{array}$ & $\begin{array}{l}\text { WG low } \\
\text { intake }\end{array}$ & $\begin{array}{l}\text { WG high } \\
\text { intake }\end{array}$ & $\begin{array}{l}\text { BMI low } \\
\text { WG } \\
\left(\mathrm{kg} / \mathrm{m}^{2}\right)\end{array}$ & $\begin{array}{l}\text { BMI high } \\
\text { WG } \\
\left(\mathrm{kg} / \mathrm{m}^{2}\right)\end{array}$ & $\begin{array}{l}\text { WCm/ } \\
\text { WHR } \\
\text { low WG }\end{array}$ & $\begin{array}{l}\text { WCm/ } \\
\text { WHR } \\
\text { high WG }\end{array}$ & $\begin{array}{l}\mathrm{Wt}, \\
\mathrm{Ht}^{*}\end{array}$ & Adjusted fort \\
\hline \multirow[t]{2}{*}{$\begin{array}{l}\text { Thane } \\
\text { et al. }{ }^{(35)}\end{array}$} & $\begin{array}{l}M / F \\
16-64\end{array}$ & $\begin{array}{l}\text { UK NDNS } \\
1986 / 87\end{array}$ & $\begin{array}{l}1041(M) \\
1045(F)\end{array}$ & $\begin{array}{l}\text { Analysis of two } \\
\text { distinct surveys - } \\
\text { NDNS } 1986 / 87 \text {, } \\
\text { base } 2197 \& \\
\text { NDNS } 2000 / 01 \text {, } \\
\text { base } 1724\end{array}$ & 5 groups & $\geq 10 \% W G$ & $7 \mathrm{~d}$ food diary & $\begin{array}{l}0 \\
0\end{array}$ & $\begin{array}{l}>48 \\
>48\end{array}$ & $\begin{array}{l}25 \cdot 1 \\
24.8\end{array}$ & $\begin{array}{l}24.9 \\
24.4\end{array}$ & & & $\begin{array}{l}1 \\
1\end{array}$ & $\begin{array}{l}\text { A, Occ, S, SES, } \\
\text { Region, Lifestyle, } \\
\text { Season }\end{array}$ \\
\hline & & $\begin{array}{l}\text { UK NDNS } \\
2000 / 01\end{array}$ & $\begin{array}{l}758(\mathrm{M}) \\
934(\mathrm{~F})\end{array}$ & & 5 groups & $\geq 10 \% W G$ & & $\begin{array}{l}0 \\
0\end{array}$ & $\begin{array}{l}>48 \\
>48\end{array}$ & $\begin{array}{l}27 \cdot 3 \\
26 \cdot 5\end{array}$ & $\begin{array}{l}27 \cdot 5 \\
27 \cdot 3\end{array}$ & $\begin{array}{l}0.96 \mathrm{~m} /- \\
0.84 \mathrm{~m} /-\end{array}$ & $\begin{array}{l}0.97 \mathrm{~m} /- \\
0.83 \mathrm{~m} /-\end{array}$ & $\begin{array}{l}1 \\
1\end{array}$ & $\begin{array}{l}\text { All above with } \\
\text { adjustment for } \\
\text { under-reporting }\end{array}$ \\
\hline $\begin{array}{l}\text { Sahyoun } \\
\text { et al. }{ }^{(21)}\end{array}$ & $\begin{array}{l}\mathrm{M} / \mathrm{F} \\
60-98\end{array}$ & USA & $\begin{array}{l}179(\mathrm{M}), \\
356(\mathrm{~F})\end{array}$ & $\begin{array}{l}4747 \text { community } \\
\text { persons }>60 \text { years } \\
\text { from Boston MA, } \\
\text { base } 1981\end{array}$ & Quartiles & $\begin{array}{l}\text { WG by USDA } \\
\text { food survey; } \\
\text { servings }\end{array}$ & $\begin{array}{l}3 \mathrm{~d} \text { food } \\
\text { record }\end{array}$ & 0.31 & 2.9 & 26.4 & 25.2 & & & 1 & $\begin{array}{l}\text { A, AA, BMI, E, } \\
\text { Edu, G, MS, PA, } \\
\text { RG, SF, LipMed }\end{array}$ \\
\hline $\begin{array}{l}\text { Bazzano } \\
\text { et al. (22) }\end{array}$ & $\begin{array}{l}\mathrm{M} \\
40-84\end{array}$ & USA & $\begin{array}{l}17881 \\
(\mathrm{M})\end{array}$ & $\begin{array}{l}\text { PHS, base } \\
22071 \text { in } 1982\end{array}$ & 4 groups & $\begin{array}{l}\text { WG in breakfast } \\
\text { cereals by } \\
\text { Jacobs } \geq 25 \% \text {; } \\
\text { servings }\end{array}$ & $\begin{array}{l}61 \text { foods } \\
\text { semi-quant } \\
\text { FFQ }\end{array}$ & 0 & $>1$ & 24.8 & 24.1 & & & $\mathrm{~s}$ & A \\
\hline $\begin{array}{l}\text { Esmaillzadeh } \\
\text { et al. }{ }^{(23)}\end{array}$ & $\begin{array}{l}\mathrm{M} / \mathrm{F} \\
18-74\end{array}$ & Iran & $\begin{array}{l}357(\mathrm{M}) \\
470(\mathrm{~F})\end{array}$ & $\begin{array}{l}\text { TLGS, base } 861 \\
\text { at screening }\end{array}$ & Quartiles & $\begin{array}{l}\text { WG by Jacobs } \\
\geq 25 \% \text {, WG } \\
\text { foods; grams }\end{array}$ & $\mathrm{FFQ}$ & 6 & 229 & $26 \cdot 4$ & 24.7 & $0.88 \mathrm{~m} / 0.89$ & $0.83 \mathrm{~m} / 0.84$ & 1 & $A, G, E$ \\
\hline $\begin{array}{l}\text { Larsson } \\
\text { et al. }{ }^{(24)}\end{array}$ & $\begin{array}{l}F, \\
40-76\end{array}$ & Sweden & $\begin{array}{l}61433 \\
(\mathrm{~F})\end{array}$ & $\begin{array}{l}\text { Swedish } \\
\text { Mammograph } \\
\text { Cohort established } \\
1987-1990 \\
\text { base } 66651\end{array}$ & 5 groups & $\begin{array}{l}\text { WG in bread } \\
\text { and breakfast } \\
\text { cereals; } \\
\text { servings }\end{array}$ & $\begin{array}{l}\text { 67-item } \\
\text { semi-quant } \\
\text { FFQ }\end{array}$ & 1.1 & 5 & 25.0 & 24.2 & & & $\mathrm{Ng}$ & $A, E$ \\
\hline $\begin{array}{l}\text { Steffen } \\
\text { et al. }{ }^{(25)} \text {; } \\
\text { see also } \\
\text { Pereira } \\
\text { et al. }{ }^{(36)}\end{array}$ & $\begin{array}{l}\mathrm{M} / \mathrm{F} \\
18-30\end{array}$ & USA & $\begin{array}{l}4304 \\
(\mathrm{M} / \mathrm{F})\end{array}$ & $\begin{array}{l}\text { CARDIA study, } \\
\text { base } 5115 \text { in } \\
\text { 1985/86. Associations } \\
\text { of plant food, dairy } \\
\text { product and meat } \\
\text { intakes with } 15 \text {-year } \\
\text { incidence BP }\end{array}$ & Quintiles & $\begin{array}{l}\text { WG intake } \\
\text { times per day; } \\
\text { for WC intake is } \\
\text { highest intake of } \\
\text { plant food }\end{array}$ & $\begin{array}{l}\text { Diet interview } \\
\text { baseline is a } \\
\text { mean of years } \\
0+7 \text {, BMI year } \\
7 \text { data }\end{array}$ & $<0.4$ & $>1.9$ & 27.3 & $26 \cdot 1$ & $0.82 \mathrm{~m} /-$ & $0.77 \mathrm{~m} /-$ & 1 & $\begin{array}{l}\text { A, G, E, RG, } \\
\text { Centre, Edu }\end{array}$ \\
\hline $\begin{array}{l}\text { Good } \\
\text { et al. }{ }^{(34)}\end{array}$ & $\begin{array}{l}\mathrm{M} / \mathrm{F} \\
19+\end{array}$ & USA & $\begin{array}{l}4776(\mathrm{M}) \\
4475(\mathrm{~F})\end{array}$ & $\begin{array}{l}\text { USDA CSFII } \\
\text { pyramid servings } \\
\text { database } \\
\text { 1994-96 }\end{array}$ & 5 groups & $\begin{array}{l}\text { WG }>51 \% \\
\text { servings }\end{array}$ & $\begin{array}{l}2 \mathrm{~d} \text { food } \\
\text { intake }\end{array}$ & $\begin{array}{l}0 \\
0\end{array}$ & $\begin{array}{l}>3 \\
>3\end{array}$ & $\begin{array}{l}26 \cdot 3 \\
26 \cdot 4\end{array}$ & $\begin{array}{l}25 \cdot 7 \\
24 \cdot 2\end{array}$ & & & $\begin{array}{l}\mathrm{Ng} \\
\mathrm{Ng}\end{array}$ & $\begin{array}{l}\text { A, E } \\
A, S, S E S\end{array}$ \\
\hline $\begin{array}{l}\text { Koh-Banerjee } \\
\text { et al. }{ }^{(26)}\end{array}$ & $\begin{array}{l}\mathrm{M} \\
40-75\end{array}$ & USA & $\begin{array}{l}27082 \\
(\mathrm{M})\end{array}$ & $\begin{array}{l}\text { HPFS, base } \\
51529 \text { in } 1986 \text {, } \\
\text { made up of dentists, } \\
\text { vets, pharmacists, } \\
\text { etc.; data reported } \\
\text { for } 1994\end{array}$ & Quintiles & $\begin{array}{l}\text { WG by FDA, } \\
\text { Jacobs } \geq 25 \% \text {, } \\
\text { and all WG } \\
\text { foods; grams }\end{array}$ & $\begin{array}{l}\text { 168-item } \\
\text { semi-quant } \\
\text { FFQ }\end{array}$ & 5.9 & 45.8 & $26 \cdot 2$ & 25.7 & & & $\mathrm{~s}$ & $\begin{array}{l}\text { A, AA, E, Fa, } \\
\text { PA, S, RG, Wt }\end{array}$ \\
\hline
\end{tabular}




\begin{tabular}{|c|c|c|c|c|c|c|c|c|c|c|c|c|c|c|c|}
\hline Study & $\begin{array}{l}\text { Sex, Age } \\
\text { range } \\
\text { (years) }\end{array}$ & Country & $n$ & Trial description & $\begin{array}{l}\text { No data } \\
\text { group }\end{array}$ & WG definition & $\begin{array}{l}\text { WG intake } \\
\text { determination }\end{array}$ & $\begin{array}{l}\text { WG low } \\
\text { intake }\end{array}$ & $\begin{array}{l}\text { WG high } \\
\text { intake }\end{array}$ & $\begin{array}{l}\text { BMI low } \\
\text { WG } \\
\left(\mathrm{kg} / \mathrm{m}^{2}\right)\end{array}$ & $\begin{array}{l}\text { BMI high } \\
\text { WG } \\
\left(\mathrm{kg} / \mathrm{m}^{2}\right)\end{array}$ & $\begin{array}{l}\text { WCm/ } \\
\text { WHR } \\
\text { low WG }\end{array}$ & $\begin{array}{l}\text { WCm/ } \\
\text { WHR } \\
\text { high WG }\end{array}$ & $\begin{array}{l}\mathrm{Wt}, \\
\mathrm{Ht}^{\star}\end{array}$ & Adjusted fort \\
\hline Liu et al. ${ }^{(27)}$ & $\begin{array}{l}F \\
38-63\end{array}$ & USA & $\begin{array}{l}74091 \\
(\mathrm{~F})\end{array}$ & $\begin{array}{l}\text { NHS,1984 base } \\
81757\end{array}$ & Quintiles & $\begin{array}{l}\text { WG by FDA, } \\
\text { Jacobs; } \\
\text { servings }\end{array}$ & $\begin{array}{l}\text { 126-item } \\
\text { semi-quant } \\
\text { FFQ }\end{array}$ & 0.6 & 3.1 & 24.9 & 24.5 & & & s & $\begin{array}{l}\text { A, AA, BMI, C, } \\
\text { E, Fa, HRT, } \\
\text { PA, S }\end{array}$ \\
\hline $\begin{array}{l}\text { Montonen } \\
\text { et al. }\end{array}$ & $\begin{array}{l}\mathrm{M} / \mathrm{F} \\
40-69\end{array}$ & Finland & $\begin{array}{l}2286(\mathrm{M}), \\
2030(\mathrm{~F})\end{array}$ & $\begin{array}{l}\text { Finnish Mobile } \\
\text { Clinical Health } \\
\text { Examination Survey } \\
\text { in } 1966-72 \text { baseline } \\
4316\end{array}$ & Quartiles & $\begin{array}{l}\text { WG by Jacobs } \\
\geq 25 \% \text {, mainly } \\
\text { rye bread, foods } \\
\text { containing rye, } \\
\text { flour, crispbread } \\
\text { etc. and breakfast } \\
\text { cereals, WG } \\
\text { foods; grams }\end{array}$ & $\begin{array}{l}\text { Diet history } \\
\text { interview over } \\
100 \text { items }\end{array}$ & 79 & 302 & 26.7 & 26.4 & & & I & $A, E, S$ \\
\hline $\begin{array}{l}\text { Steffen } \\
\text { et al. }{ }^{(29)}\end{array}$ & $\begin{array}{l}\mathrm{M} / \mathrm{F} \\
13 / 12 \cdot 9\end{array}$ & USA & $\begin{array}{l}155(\mathrm{~B}) \\
130(\mathrm{G})\end{array}$ & $\begin{array}{l}\text { Minneapolis } \\
\text { students aged } \\
13-15,357 \text { screened, } \\
\text { baseline } 285 \text {, who } \\
\text { completed } 2 \text { insulin } \\
\text { clamp studies }\end{array}$ & Tertiles & $\begin{array}{l}\text { WG Jacobs, } \\
\geq 25 \% \text {; servings }\end{array}$ & $\begin{array}{l}\text { 127-item } \\
\text { semi-quant } \\
\text { FFQ }\end{array}$ & 0.4 & 2.6 & 23.6 & $21 \cdot 9$ & $0.814 \mathrm{~m} /-$ & $0.768 \mathrm{~m} /-$ & 1 & $\begin{array}{l}\text { A, E, G, R, } \\
\text { Tanner stage }\end{array}$ \\
\hline $\begin{array}{l}\text { Steffen } \\
\text { et al. }{ }^{(30)}\end{array}$ & $\begin{array}{l}\mathrm{M} / \mathrm{F} \\
45-64\end{array}$ & USA & $\begin{array}{l}4083 \\
(\text { w M), } \\
4755 \\
(\text { w F), } \\
1188 \\
\text { (AA M), } \\
1915 \text { (AA F) }\end{array}$ & $\begin{array}{l}\text { ARIC study, } \\
\text { Minneapolis, at } \\
\text { screening } 15792 \\
\text { ) }\end{array}$ & Quintiles & $\begin{array}{l}\text { WG by Jacobs } \\
\geq 25 \% \text {; servings }\end{array}$ & $\begin{array}{l}\text { 66-item } \\
\text { semi-quant } \\
\text { FFQ }\end{array}$ & 0.1 & 3 & $27 \cdot 2$ & $27 \cdot 3$ & $-/ 0.928$ & $-/ 0.912$ & $\mathrm{Ng}$ & $A, E, G, R$ \\
\hline $\begin{array}{l}\text { McKeown } \\
\text { et al. }{ }^{(31)}\end{array}$ & $\begin{array}{l}\mathrm{M} / \mathrm{F} \\
54\end{array}$ & USA & $\begin{array}{l}1338(\mathrm{M}) \\
1603(\mathrm{~F})\end{array}$ & $\begin{array}{l}\text { Framingham } \\
\text { Offspring Study, } \\
3799 \text { at } 5 \text { th } \\
\text { examination cycle } \\
1991-1995\end{array}$ & Quintiles & $\begin{array}{l}\text { WG by Jacobs } \\
\geq 25 \% \text {; servings }\end{array}$ & $\begin{array}{l}\text { 126-item } \\
\text { semi-quant } \\
\text { FFQ }\end{array}$ & 0.13 & $2 \cdot 9$ & $26 \cdot 9$ & 26.4 & $\begin{array}{l}0.932 \mathrm{~m} / \\
0.92\end{array}$ & $\begin{array}{l}0.912 \mathrm{~m} / \\
0.91\end{array}$ & 1 & $\begin{array}{l}\text { A, AA, BMI, E, } \\
\text { F, Fa, G, HRT, } \\
\text { PA, PF, S, V }\end{array}$ \\
\hline \multirow[t]{2}{*}{$\begin{array}{l}\text { Jacobs } \\
\text { et al. }{ }^{(32)}\end{array}$} & $\begin{array}{l}\mathrm{M}, \\
35-56\end{array}$ & Norway & $\begin{array}{l}16933 \\
(\mathrm{M})\end{array}$ & $\begin{array}{l}\text { Norwegian County } \\
\text { Study, } 41174 \text { at }\end{array}$ & 5 groups & $\begin{array}{l}\text { WG in whole- } \\
\text { grain bread only; }\end{array}$ & 66-item FFQ & $0.05-0.6$ & $2 \cdot 25-5 \cdot 40$ & $25 \cdot 3$ & $25 \cdot 2$ & & & $\mathrm{Ng}$ & $A, E$ \\
\hline & $\begin{array}{l}\mathrm{F}, \\
35-56\end{array}$ & Norway & $\begin{array}{l}16915 \\
(\mathrm{~F})\end{array}$ & $\begin{array}{l}\text { systematic base } \\
\text { screening, 1977-1983 }\end{array}$ & 5 groups & score given & 66-item FFQ & $0.05-0.6$ & $2 \cdot 25-5 \cdot 40$ & 24.6 & 24.5 & & & $\mathrm{Ng}$ & $A, E$ \\
\hline $\begin{array}{l}\text { Jacobs } \\
\text { et al. }{ }^{(33)}\end{array}$ & $\begin{array}{l}\mathrm{F}, \\
55-69\end{array}$ & USA & $\begin{array}{l}34492 \\
(F)\end{array}$ & $\begin{array}{l}\text { IWHS, } 41836 \text { at } \\
\text { screening }\end{array}$ & Quintiles & $\begin{array}{l}\text { WG by Jacobs } \\
\geq 25 \% \text {; servings }\end{array}$ & $\begin{array}{l}\text { 127-item } \\
\text { semi-quant } \\
\text { FFQ }\end{array}$ & 0.21 & 3.2 & $27 \cdot 2$ & $26 \cdot 9$ & $-/ 0 \cdot 848$ & $-/ 0.832$ & s & $\begin{array}{l}\text { A, E (BMI in } \\
\text { WHR only) }\end{array}$ \\
\hline
\end{tabular}

WG, whole-grain/whole grains; BMI, body mass index; WCm, waist circumference in metres; WHR, waist:hip ratio; Wt, weight; Ht, height; M, males; F, females; NDNS, National Diet and Nutrition Survey; B, boys; G, girls; w, White; AA, African American; base, baseline; PHS, Physicians' Health Study; TLGS, Tehran Lipid and Glucose Study; BP, blood pressure; CARDIA, Coronary Artery Risk Development in Young Adults; USDA, US Department of Agriculture; CSFI, Continuing Survey of Food Intakes by Individuals; HPFS, Health Professionals' Follow-Up Study; NHS, Nurses' Health Study; ARIC, Atherosclerosis Risk in Community; IWHS, Iowa Women's Health Study; FDA, Food and Drug Administration; semi-quant, semi-quantitative; FFQ, food-frequency questionnaire.

* Method used to determine Wt and height $\mathrm{Ht}$. measured independently (I), by self (S) or information not given ( $\mathrm{Ng}$ ).

t Data adjusted for following main factors. age (A), alcohol intake (AA), BMl, caffeine (C), dieting and eating behaviour (DE), dietary fibre intake (DF), disease history (DH), energy intake (E), education (Edu), specific foods $(\mathrm{F})$, fat intake $(\mathrm{Fa})$, gender $(\mathrm{G})$, hypertension $(\mathrm{H})$, hormone replacement therapy status (HRT), lipid-lowering or hypertensive medication (LipMed), marital status (MS), occupation (Occ), physical activity (PA), polyunsaturated fat intake (PF), race (R), refined grain intake at base and changes (RG), smoking (S), socio-economic status (SES), saturated fat intake (SF), multivitamin use (V), WC and Wt. 


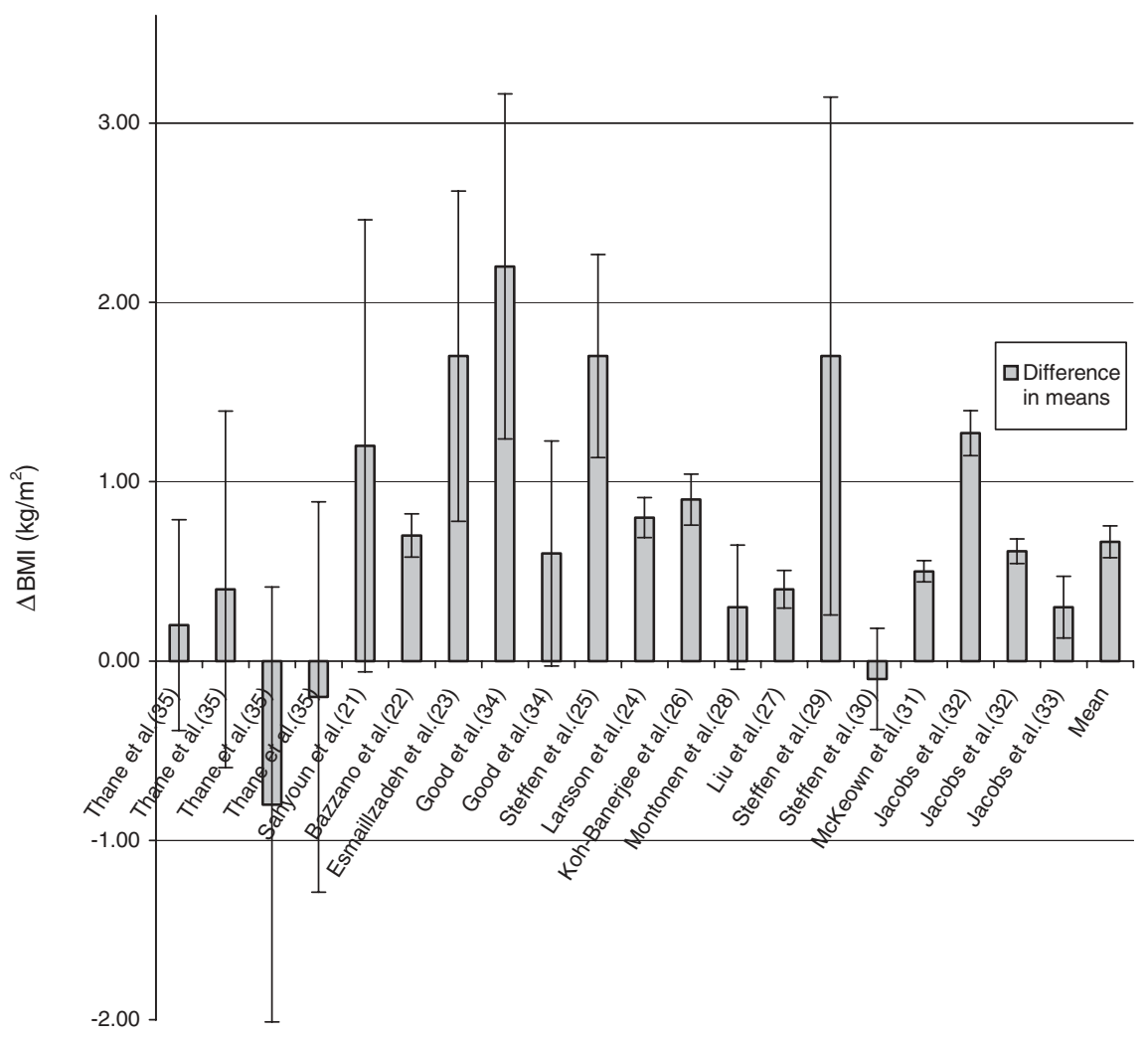

Fig. 2 Mean difference in body mass index ( $\triangle \mathrm{BMI})$ in non- or low consumers of whole grains compared with high consumers of whole grains ( $95 \%$ confidence interval shown by vertical bars)

\begin{tabular}{|c|c|c|c|c|c|c|}
\hline \multirow[t]{2}{*}{ Study name } & \multirow[t]{2}{*}{ Subgroup within study } & \multicolumn{5}{|c|}{ Statistics for each study } \\
\hline & & $\begin{array}{l}\text { Difference } \\
\text { in means }\end{array}$ & $\begin{array}{l}\text { Standard } \\
\text { error }\end{array}$ & $\begin{array}{l}\text { Lower } \\
\text { limit }\end{array}$ & $\begin{array}{c}\text { Upper } \\
\text { limit }\end{array}$ & $P$ value \\
\hline Esmaillzadeh et al.(23) & WHR & 0.050 & 0.008 & 0.034 & 0.066 & 0.000 \\
\hline Jacobs et al.(33) & WHR & 0.016 & 0.001 & 0.013 & 0.019 & 0.000 \\
\hline McKeown et al.(31) & WHR & 0.030 & 0.006 & 0.018 & 0.042 & 0.000 \\
\hline Steffen et al.(30) & WHR & 0.016 & 0.001 & 0.013 & 0.019 & 0.000 \\
\hline Mean random-effects & s model & 0.023 & 0.003 & 0.016 & 0.030 & 0.000 \\
\hline Esmaillzadeh et al.(23) & WC & 0.050 & 0.001 & 0.048 & 0.052 & 0.000 \\
\hline McKeown et al.(31) & WC & 0.020 & 0.008 & 0.005 & 0.035 & 0.009 \\
\hline Steffen et al.(29) & WC & 0.046 & 0.017 & 0.013 & 0.079 & 0.006 \\
\hline Steffen et al.(25) & WC & 0.050 & 0.006 & 0.039 & 0.061 & 0.000 \\
\hline Thane et al.(35) M & WC & 0.010 & 0.001 & 0.008 & 0.012 & 0.000 \\
\hline Thane et al.(35) F & WC & -0.010 & 0.001 & -0.012 & -0.008 & 0.000 \\
\hline Mean random-effects & s model & 0.027 & 0.013 & 0.002 & 0.052 & 0.033 \\
\hline
\end{tabular}

intake led to increased energy $(1.80 \mathrm{MJ}(431 \mathrm{kcal})$, $P<0.05)$ and dietary fibre intake $(9 \mathrm{~g}, P<0.01)$. Total and saturated fat intakes were reduced non-significantly by $10.1 \mathrm{~g}$ and $3.8 \mathrm{~g}$ respectively in the high intake group. Other aspects of healthy lifestyle that were reported in a consistent format were alcohol intake and number of smokers, which were both lower in the high whole-grain whole-grain intake is compared with high intake
Difference in means and $95 \% \mathrm{Cl}$ $\mid$

$-0.25$

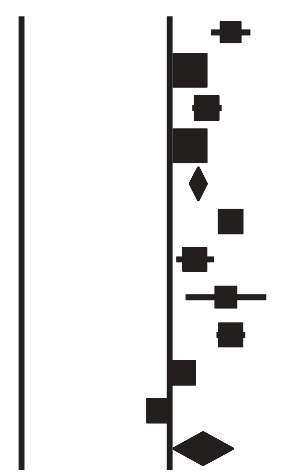

0.00

Fig. 3 Mean difference (and $95 \%$ confidence interval, $\mathrm{Cl}$ ) in waist:hip ratio (WHR) or waist circumference (WC) when low or no

group, significantly so in the case of smokers, where there were $12.4 \%$ fewer $(P<0 \cdot 01)$. The proportion undertaking regular physical activity was increased by $11.4 \%$ in the high whole-grain group, but this did not reach significance. Data relating to micronutrient intake or mineral supplementation were not reported with sufficient consistency to be collated, but where reported was generally 
Table 2 Subgroup analysis of studies resulting from this systematic review

\begin{tabular}{lccccc}
\hline Factor & No. of data pairs & No. of subjects & $\begin{array}{c}\text { Mean difference } \\
\text { in BMl }\left(\mathrm{kg} / \mathrm{m}^{2}\right)\end{array}$ & \multicolumn{1}{c}{$\begin{array}{c}\text { Significance, } \\
P\end{array}$} \\
\hline All & 20 & 119829 & 0.630 & $0.460,0.800$ & $<0.0001$ \\
Studies with no dietary data excluded & 17 & 91910 & 0.571 & $0.360,0.762$ & $<0.0001$ \\
Studies with less than about three servings per day & 17 & 104708 & 0.579 & $0.382,0.776$ & $<0.0001$ \\
Gender & & & & & \\
$\quad$ Males & 6 & 34075 & 0.671 & $0.505,0.837$ & $<0.0001$ \\
$\quad$ Females & 7 & 75059 & 0.695 & $0.371,1.019$ & $<0.0001$ \\
Location & 11 & 78662 & 0.645 & $0.417,0.873$ & $<0.0001$ \\
$\quad$ North America & 8 & 40721 & 0.570 & $0.305,0.834$ & $<0.0001$ \\
$\quad$ Europe & & & &
\end{tabular}

$\mathrm{BMI}$, body mass index; $\mathrm{Cl}$, confidence interval.

Table 3 The associations between high or low intake of whole grains and mean energy, fat, saturated fat and fibre intake and various lifestyle factors

\begin{tabular}{|c|c|c|c|c|c|c|c|c|}
\hline & \multicolumn{2}{|c|}{ Energy } & \multirow[b]{2}{*}{ Fat $(g / d)$} & \multirow{2}{*}{$\begin{array}{l}\text { Saturated } \\
\text { fat }(g / d)\end{array}$} & \multirow{2}{*}{$\begin{array}{l}\text { Fibre } \\
\text { (g/d) }\end{array}$} & \multirow{2}{*}{$\begin{array}{c}\text { Alcohol } \\
(\mathrm{g} / \mathrm{d})\end{array}$} & \multirow{2}{*}{$\begin{array}{c}\text { Smokers } \\
(\%)\end{array}$} & \multirow{2}{*}{$\begin{array}{c}\text { Regular } \\
\text { exercise (\% }\end{array}$} \\
\hline & $(\mathrm{MJ} / \mathrm{d})$ & $(\mathrm{kcal} / \mathrm{d})$ & & & & & & \\
\hline No. of studies & \multicolumn{2}{|c|}{17} & 8 & 9 & 9 & 5 & 12 & 6 \\
\hline None or low whole-grain intake & 7.427 & $1775 \cdot 1$ & $86 \cdot 2$ & $32 \cdot 0$ & 14.7 & $10 \cdot 2$ & 29.8 & $40 \cdot 1$ \\
\hline High whole-grain intake & 9.229 & 2205.7 & $76 \cdot 1$ & $28 \cdot 2$ & 23.7 & $6 \cdot \overline{3}$ & $17 \cdot 4$ & 51.5 \\
\hline SED & 0.984 & 235.1 & $20 \cdot 2$ & $6 \cdot 6$ & 4.4 & 3.3 & 4.0 & $18 \cdot 4$ \\
\hline Significance, $P$ & \multicolumn{2}{|c|}{$<0.05$} & NS & NS & $<0.01$ & NS & 0.02 & NS \\
\hline
\end{tabular}

SED, standard error of the difference; NS, not significant.

higher or supplementation more prevalent in the higher whole-grain group.

\section{Discussion}

Epidemiological data have been used to suggest that the consumption of at least one serving of whole-grain foods each day is associated with a reduced risk of death from cardiovascular disease $\mathrm{s}^{(32,33,37,38)}$ and reduced incidence of diabetes ${ }^{(28,39)}$ and the metabolic syndrome ${ }^{(23,40)}$. Obesity is a risk factor for all of these conditions; hence it is perhaps not surprising that in this, the most complete systematic review of the epidemiological evidence reported to date, an association has been demonstrated between a higher whole-grain intake and BMI. This association is in agreement with the findings from two large cohort studies, the Nurses' Study and Health Professionals' Follow-Up Study ${ }^{(26,27)}$, both of which reported a reduction in weight gain over at least 7 years when whole-grain intake was about three servings per day. In addition there is limited evidence provided by short-term clinical trials ${ }^{(41,42)}$ that whole grains, when included in an energy-restricted diet, were at least as effective as other regimens.

The reduction reported in the subgroup analysis with about three whole-grain servings is $0.58 \mathrm{~kg} / \mathrm{m}^{2}$ for $\mathrm{BMI}$; waist circumference is about $2.7 \mathrm{~cm}$ less, equivalent to a reduction of approximately $3 \%$ assuming an average waist circumference of $90 \mathrm{~cm}$. While this reduction may in itself be quite modest, the healthy lifestyle factors (lower prevalence of smoking and greater participation in physical activity) associated with higher whole-grain consumption and the dietary changes (reduction in total and saturated fat, increased fibre intake and moderate alcohol consumption) add strength to public health messages encouraging its intake.

A further aspect that has previously been reported with reference to the NDNS 2000/01 is a greater micronutrient density in those consuming more whole-grain foods (Thane et al., personal communication).

Overall the findings are very much in line with anthropometric changes in adults reported when factor analysis of food patterns were used to predict BW and adiposity in the Baltimore Longitudinal Study of $\operatorname{Aging}^{(43)}$.

The potential mechanism(s) by which whole grains result in a healthier weight is speculative. In both clinical trials and observational studies the intake of whole grains has been inversely associated with plasma biomarkers of obesity including insulin, C-peptide and leptin concentration $^{(44)}$. The key mechanisms involved appear to relate to the role that whole grains have on satiety and insulin sensitivity, and as a source of dietary fibre and antioxidants. It is speculated ${ }^{(11)}$ that whole grains or their components may influence hormonal factors; whether the active moiety is fibre or one of the micro-components, such as lignan or phytosterol, is not clear. 


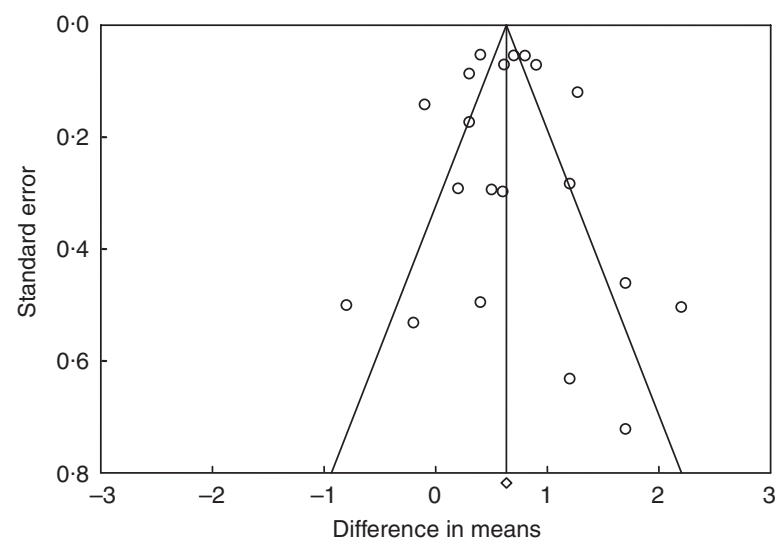

Fig. 4 Funnel plot of standard error by difference in means

It has been suggested that there is no real mechanism of action and whole-grain consumption is simply a marker of a healthier lifestyle - that the benefits reported in epidemiological studies relate to the lower fat, higher carbohydrate and higher fibre intakes reported. However, this argument is not entirely plausible. Certainly reported intake of fruits and vegetables is generally higher and meat consumption lower in diets rich in whole grains, factors that would generally be considered beneficial to health and weight management ${ }^{(43)}$. However, when data from the Framingham Offspring Cohort ${ }^{(31)}$ were adjusted for potential confounding variables and dietary factors associated with diets high in whole grains, the association between whole-grain intake and BMI or WHR - while attenuated - was still significant.

One of the main weaknesses of this analysis is the reliance on observational data. The unreliability of FFQs to determine food intake, in particular whole-grain foods which have been defined inconsistently over the years, does not enhance this case. In addition, half of the studies used in this analysis have relied on self-reported measures of height and weight. All studies cited have made some adjustment to the data, primarily age, gender and energy intake, but again the degree of sophistication of the models employed is highly variable.

A funnel plot (Fig. 4) was used to test for publication bias; study size (as measured by standard error) was plotted as a function of effect size. Generally, large studies appear towards the top of the plot and tend to cluster near the mean effect size. Smaller studies appear towards the bottom of the plot, and (since there is more sampling variation in effect size estimates in the smaller studies) will be dispersed across a range of values. As the studies appear to be distributed symmetrically about the combined effect size an absence of publication bias is suggested.

Despite these various shortcomings the association between whole-grain intake and lower BW is highly significant $(P<0.0001)$ and is the result of the integration of a large number (20) of data pairs including about 120000 subjects. The consumption of about three daily servings of whole grains is associated with lower BMI and central adiposity. In addition, people who consume more whole grains are likely to have a healthier lifestyle as fewer of them smoke, they exercise more frequently and they tend to have lower fat and higher fibre intake. The evidence identified supports the inclusion of whole grains in diets for the maintenance of healthy body weight and indicates it can be promoted as a marker of healthy body weight.

\section{Acknowledgements}

Provision of additional data by Drs Larsson, Liu, Jacobs, McKeown and Rimm is gratefully acknowledged, as is permission to use data from the Framingham Offspring Cohort.

\section{References}

1. World Health Organization (2000) Obesity: Preventing and Managing the Global Epidemic. WHO Technical Report Series no. 894. Geneva: WHO.

2. Department of Health (2005) Healthier England. London: The Stationery Office.

3. National Institute for Health and Clinical Excellence (2006) Obesity: Guidance on the Prevention, Identification and Management of Obesity and Overweight in Adults and Children. NICE Clinical Publication no. 43. London: NICE; available at http://www.nice.org.uk/CG043

4. US Department of Agriculture (2005) Dietary Guidelines for Americans 2005. http://www.health.gov/dietaryguidelines/ dga2005/recommendations.htm (accessed January 2007).

5. World Health Organization (2003) Recommendations for preventing excess weight gain and obesity. In Diet, Nutrition and the Prevention of Chronic Diseases. Joint WHO/FAO Expert Consultation. WHO Technical Report Series no. 916, pp. 61-71. Geneva: WHO; available at http://whqlibdoc.who.int/trs/WHO_TRS_916.pdf

6. Pereira MA \& Ludwig DS (2001) Dietary fiber and bodyweight regulation. Observations and mechanisms. Pediatr Clin North Am 48, 969-980.

7. Howarth NC, Saltzman E \& Roberts SB (2001) Dietary fiber and weight regulation. Nutr Rev 59, 129-139.

8. Seal CJ (2006) Whole grains and CVD risk. Proc Nutr Soc 65, 24-34

9. Slavin J (2003) Why whole grains are protective: biological mechanisms. Proc Nutr Soc 62, 129-134.

10. Anderson JW (2003) Whole grains protect against atherosclerotic cardiovascular disease. Proc Nutr Soc 62, 135-142.

11. Koh-Banerjee P \& Rimm EB (2003) Whole grain consumption and weight gain: a review of the epidemiological evidence, potential mechanisms and opportunities for future research. Proc Nutr Soc 62, 25-29.

12. Food and Drug Administration (1999) Whole-Grain Foods Authoritative Statement Claim, Notification Docket 99P-2209. http://www.cfsan.fda.gov/wdms/flg.6c.html (accessed June 2007).

13. Richardson DP (2003) Wholegrain health claims in Europe. Proc Nutr Soc 62, 161-169.

14. Stroup DF, Berlin JA, Morton SC, Olkin I, Williamson GD, Rennie D, Moher D, Becker BJ, Sipe TA \& Thacker SB (2000) Meta-analysis of observational studies in epidemiology: a proposal for reporting. Meta-analysis of 
Observational Studies in Epidemiology (MOOSE) group. JAMA 283, 2008-2012.

15. Jacobs Jr DR, Marquart L, Slavin J \& Kushi LH (1998) Whole-grain intake and cancer: an expanded review and meta-analysis. Nutr Cancer 30, 85-96.

16. Thane CW, Jones AR, Stephen AM, Seal CJ \& Jebb SA (2005) Whole-grain intake of British young people aged 4-18 years. BrJ Nutr 94, 825-831.

17. Lang R, Thane CW, Bolton-Smith C \& Jebb SA (2003) Consumption of whole-grain foods by British adults: findings from further analysis of two national dietary surveys. Public Health Nutr 6, 479-484.

18. Lang R \& Jebb SA (2003) Who consumes whole grains, and how much? Proc Nutr Soc 62, 123-127.

19. Cleveland LE, Moshfegh AJ, Albertson AM \& Goldman JD (2000) Dietary intake of whole grains. J Am Coll Nutr 19, 331S-338S.

20. Lean ME, Han TS \& Seidell JC (1998) Impairment of health and quality of life in people with large waist circumference. Lancet 351, 853-856.

21. Sahyoun NR, Jacques PF, Zhang XL, Juan W \& McKeown NM (2006) Whole-grain intake is inversely associated with the metabolic syndrome and mortality in older adults. $\mathrm{Am} \mathrm{J}$ Clin Nutr 83, 124-131.

22. Bazzano LA, Song Y, Bubes V, Good CK, Manson JE \& Liu S (2005) Dietary intake of whole and refined grain breakfast cereals and weight gain in men. Obes Res 13, 1952-1960.

23. Esmaillzadeh A, Mirmiran P \& Azizi F (2005) Whole-grain consumption and the metabolic syndrome: a favorable association in Tehranian adults. Eur J Clin Nutr 59, 353-362.

24. Larsson SC, Giovannucci E, Bergkvist L \& Wolk A (2005) Whole grain consumption and risk of colorectal cancer: a population-based cohort of 60000 women. Br J Cancer $\mathbf{9 2}$, 1803-1807.

25. Steffen LM, Kroenke CH, Yu X, Pereira MA, Slattery ML, Van Horn L, Gross MD \& Jacobs Jr DR (2005) Associations of plant food, dairy product, and meat intakes with $15-y$ incidence of elevated blood pressure in young black and white adults: the Coronary Artery Risk Development in Young Adults (CARDIA) Study. Am J Clin Nutr 82, 1169-1177.

26. Koh-Banerjee P, Franz M, Sampson L, Liu S, Jacobs Jr DR, Spiegelman D, Willett W \& Rimm E (2004) Changes in whole-grain, bran, and cereal fiber consumption in relation to 8-y weight gain among men. Am J Clin Nutr 80, $1237-1245$.

27. Liu S, Willett WC, Manson JE, Hu FB, Rosner B \& Colditz G (2003) Relation between changes in intakes of dietary fiber and grain products and changes in weight and development of obesity among middle-aged women. Am J Clin Nutr 78, 920-927.

28. Montonen J, Knekt P, Jarvinen R, Aromaa A \& Reunanen A (2003) Whole-grain and fiber intake and the incidence of type 2 diabetes. Am J Clin Nutr 77, 622-629.

29. Steffen LM, Jacobs Jr DR, Murtaugh MA, Moran A, Steinberger J, Hong CP \& Sinaiko AR (2003) Whole grain intake is associated with lower body mass and greater insulin sensitivity among adolescents. Am J Epidemiol $\mathbf{1 5 8}$, 243-250.

30. Steffen LM, Jacobs Jr DR, Stevens J, Shahar E, Carithers T \& Folsom AR (2003) Associations of whole-grain, refinedgrain, and fruit and vegetable consumption with risks of all-cause mortality and incident coronary artery disease and ischemic stroke: the Atherosclerosis Risk in Communities (ARIC) Study. Am J Clin Nutr 78, 383-390.

31. McKeown NM, Meigs JB, Liu S, Wilson PW \& Jacques PF (2002) Whole-grain intake is favorably associated with metabolic risk factors for type 2 diabetes and cardiovascular disease in the Framingham Offspring Study. Am J Clin Nutr 76, 390-398.

32. Jacobs Jr DR, Meyer HE \& Solvoll K (2001) Reduced mortality among whole grain bread eaters in men and women in the Norwegian County Study. Eur J Clin Nutr $\mathbf{5 5}$, $137-143$

33. Jacobs Jr DR, Meyer KA, Kushi LH \& Folsom AR (1998) Whole-grain intake may reduce the risk of ischemic heart disease death in postmenopausal women: the Iowa Women's Health Study. Am J Clin Nutr 68, 248-257.

34. Good CK, Holschuh K, Albertson AK \& Eldridge AK. (2004) The relationship between whole grain consumption and body mass index in adult men and women: data from the USDA's continuing survey of food intakes by individuals, pyramid servings data, 1994-1996. Presented at Experimental Biology 2004: Translating the Genome, Washington, DC, 17-21 April, FASEB006960 107.3abs.

35. Thane CW, Stephen AM \& Jebb SA (2007) Whole-grains and adiposity: little association among British adults. Eur J Clin Nutr (Epublication ahead of print version)

36. Pereira MA, Jacobs DR, Slattery ML, Ruth KJ, Van Horn L, Hilner JE \& Kushi LHl (1998) The association of whole grain intake and fasting insulin in a biracial cohort of young adults: the CARDIA study. CVD Prev 1, 233-242.

37. Jensen MK, Koh-Banerjee P, Hu FB, Franz M, Sampson L, Gronbaek M \& Rimm EB (2004) Intakes of whole grains, bran, and germ and the risk of coronary heart disease in men. Am J Clin Nutr 80, 1492-1499.

38. Liu S, Stampfer MJ, Hu FB, Giovannucci E, Rimm E, Manson JE, Hennekens CH \& Willett WC (1999) Whole-grain consumption and risk of coronary heart disease: results from the Nurses' Health Study. Am J Clin Nutr 70, 412-419.

39. Pereira MA, Jacobs Jr DR, Van Horn L, Slattery ML, Kartashov AI \& Ludwig DS (2002) Dairy consumption, obesity, and the insulin resistance syndrome in young adults: the CARDIA Study. JAMA 287, 2081-2089.

40. McKeown NM, Meigs JB, Liu S, Saltzman E, Wilson PW \& Jacques PF (2004) Carbohydrate nutrition, insulin resistance, and the prevalence of the metabolic syndrome in the Framingham Offspring Cohort. Diabetes Care 27, 538-546.

41. Melanson KJ, Angelopoulos TJ, Nguyen VT, Martini M, Zukley L, Lowndes J, Dube TJ, Fiutem JJ, Yount BW \& Rippe JM (2006) Consumption of whole-grain cereals during weight loss: effects on dietary quality, dietary fiber, magnesium, vitamin B-6, and obesity. J Am Diet Assoc 106, $1380-1388$.

42. Saltzman E, Das SK, Lichtenstein AH, Dallal GE, Corrales A, Schaefer EJ, Greenberg AS \& Roberts SB (2001) An oatcontaining hypocaloric diet reduces systolic blood pressure and improves lipid profile beyond effects of weight loss in men and women. J Nutr 131, 1465-1470.

43. Newby PK, Muller D, Hallfrisch J, Andres R \& Tucker KL (2004) Food patterns measured by factor analysis and anthropometric changes in adults. Am J Clin Nutr 80, 504-513.

44. Fung TT, Rimm EB, Spiegelman D, Rifai N, Tofler GH, Willett WC \& Hu FB (2001) Association between dietary patterns and plasma biomarkers of obesity and cardiovascular disease risk. Am J Clin Nutr 73, 61-67. 